\title{
Electromagnetic emission from magnetite plate cracking under seismic processes
}

\author{
S. Koshevaya ${ }^{1}$, V. Grimalsky ${ }^{1}$, N. Makarets $^{2}$, A. Kotsarenko ${ }^{3}$, J. Siquieros-Alatorre ${ }^{1}$, R. Perez-Enriquez ${ }^{3}$, and \\ D. Juarez-Romero ${ }^{1}$ \\ ${ }^{1}$ Autonomous University of Morelos, UAEM, Av.Universidad 1001, Col. Chamilpa, Z.P. 62209, Cuernavaca, Mor., Mexico \\ ${ }^{2}$ Department of Physics, Kyiv National Shevchenko University, Volodymyrska 64, Kyiv, Z.P. 01033, Ukraine \\ ${ }^{3}$ Centro de Geociencias, UNAM, A.P. 1-742, Querétaro, Z.P. 76001, Mexico
}

Received: 16 June 2007 - Revised: 2 September 2007 - Accepted: 3 September 2007 - Published: 2 January 2008

\begin{abstract}
Electromagnetic emission generated by cracking of a magnetite plate is theoretically investigated. The nonstationary mechanical stresses, produced by moving the tip of a crack and a wave of mechanical unloading in the plate are considered as the sources of the radiation. It is demonstrated that the radiation is produced by the appearance of a non-stationary magnetic moment in the plate.
\end{abstract}

\section{Introduction}

In the last decade many articles (see review by Hayakawa and Molchanov, 2004) have been devoted to the research of electromagnetic emission and fields, which emerge during earthquakes and volcano eruptions, and also to the development of various models of their generation. Electromagnetic fields, electronic, and lattice subsystems of a solid body play the main role in various dynamical processes. Therefore three different time scales exist in any crystal of a volume $V \approx L^{3}$ : electromagnetic $\tau_{l}=L / c_{l}$, electronic $\tau_{e}=L / c_{F}$, and the lattice $\tau_{s}=L / c_{s}$, where $c_{l, s, F}$ are velocities of light and sound, and Fermi velocity in the solid, respectively. The relations $\tau_{l} \ll \tau_{e} \ll \tau_{s}$ indicate that the frequencies of the corresponding emissions have inverse relations. The power, another important characteristic of the emission, is proportional to the geometrical measure of the source.

Thus, as a first approximation, we can classify different sources of electromagnetic emission and fields produced by a crystal fracture in two groups: 1) a type of physical process at the source; 2) a geometrical measure of the source. In this paper we are interested in the emission of electromagnetic (EM) waves from a cracked piezo-magnetic crystal. Note that the electromagnetic emission from cracking ionic non-

Correspondence to: S. V. Koshevaya

(svetlana@uaem.mx) magnetic crystals was investigated during many years (Gershenzon et al., 1986; Biadzhi et al., 1990).

\section{Basic equations and model}

Consider that the main reason for the electromagnetic wave emission is the occurrence of a piezo-crystal cracking (Gernets et al., 2002). Assume that the front of a moving crack in an unbounded brittle crystal is an infinite straight line at time $t$. Hence, the crystal is tense far before the front, and is unloaded far behind it. Assume that, due to critical external stresses, this front moves over a length $l$ along its normal line during a time $\tau$. Hence, at time $t+\tau$ the volume of the unloaded crystal part is increased by an amount $V_{\tau}$, and the volume of the tense part is decreased by the same amount.

According to Landau and Lifshitz (1982), the piezomagnetic tensor cannot vanish only for crystals with a magnetic structure. Such crystals, which are most readily found in nature, belong to the spinel group with a chemical formula $\mathrm{Me}^{2+} \mathrm{O} \cdot \mathrm{Fe}_{2}^{3+} \mathrm{O}_{3}$, where Me is a transition metal in a divalent state. According to Vainshtein (1982) and Gilder et al. (2004) these belong to the $F d 3 m$ space group of symmetry and can have different point groups of symmetry. As an example, we will treat the crystal $\mathrm{CoFe}_{2} \mathrm{O}_{4}$, whose material tensors belong to the point group $6 \mathrm{~mm}$ (see Huang et al., 1997). In this section, we assume nonmagnetic rocks with similar mechanical and dielectric properties surrounding the piezo-magnetic crystal. Therefore, we do not take into account boundary conditions for mechanical strains and EM field, and solve equations for unbounded piezo-magnetic medium. The equations of motion and of electromagnetic field in the crystallographic coordinate frame with parameters: $\rho$ is the crystal density, $\boldsymbol{U}$ is the strain vector, $\sigma_{i j}$, are mechanical stresses tensor components, $\partial_{j}$, and $\partial_{t}$ are partial derivative with respect to coordinate $x_{j}(i, j=1,2,3)$ and time, $\boldsymbol{E}, \boldsymbol{H}, \boldsymbol{D}, \boldsymbol{B}$

Published by Copernicus Publications on behalf of the European Geosciences Union. 


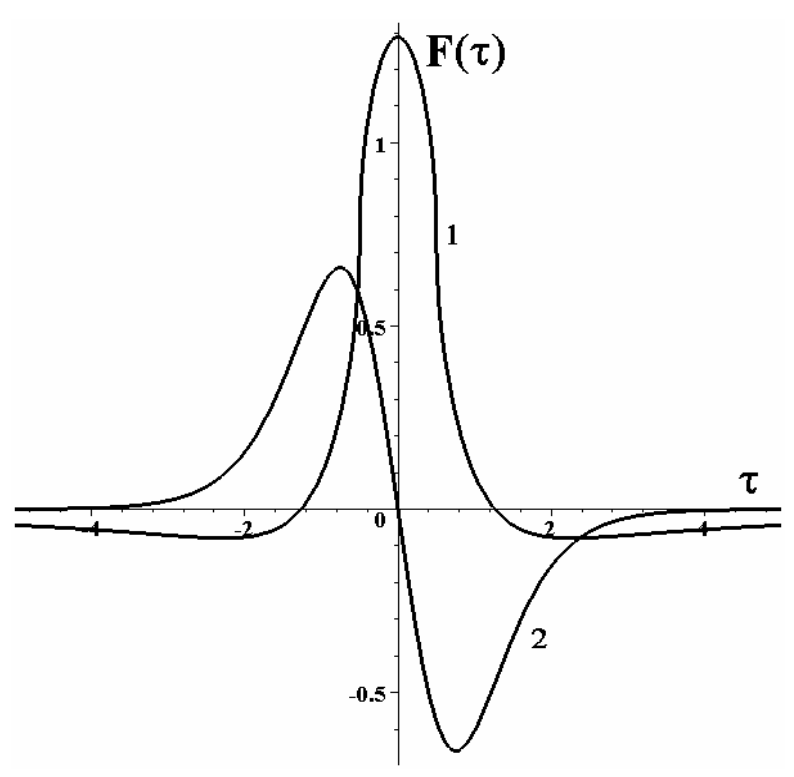

Fig. 1. Dimensionless amplitude of magnetic field emitted during cracking of a piezomagnetic crystal on dimensionless time 1) radiation generated by the tip of crack $\left.F_{t}(\tau), 2\right)$ radiation generated by the wave of unloading $F_{u}(\tau)$ at $L / a=1$.

are vectors of electric and magnetic field and inductions:

$$
\begin{aligned}
& \rho \partial_{t}^{2} U_{i}(\boldsymbol{r}, t)=\partial_{j} \sigma_{i j}(\boldsymbol{r}, t), \\
& \operatorname{rot} \boldsymbol{H}(\boldsymbol{r}, t)=\partial_{t} \boldsymbol{D}(\boldsymbol{r}, t), \\
& \operatorname{rot} \boldsymbol{E}(\boldsymbol{r}, t)=-\partial_{t} \boldsymbol{B}(\boldsymbol{r}, t), \\
& \operatorname{div} \boldsymbol{B}(\boldsymbol{r}, t)=0, \\
& \operatorname{div} \boldsymbol{D}(\boldsymbol{r}, t)=0,
\end{aligned}
$$

The constitutive equations are for the piezo-magnetic crystal described by parameters $U_{k l}=\left(\partial_{l} U_{k}+\partial_{k} U_{l}\right) / 2$ are strain tensor components, $c_{i j k l}, q_{i j k}, \mu_{i j}, \varepsilon_{i j}$ are tensor components of elastic, piezo-magnetic, magnetic, and dielectric constants of magnetite (Pan et al., 2001), $\varepsilon_{0}, \mu_{0}$ are electric and magnetic constants:

$$
\begin{aligned}
& \sigma_{i j}=c_{i j k l} U_{k l}-q_{k i j} H_{k}, \\
& B_{i}=\mu_{0} \mu_{i j} H_{j}+q_{i k l} U_{k l}, \\
& D_{i}=\varepsilon_{0} \varepsilon_{i j} E_{j},
\end{aligned}
$$

and Eqs. (1) and (2) have been solved for magnetic field only by using the Laplace transform with a parameter $p$ for the time, and the Fourier transform with a wave vector $\boldsymbol{k}$ for coordinates. After the standard transformations we have the components for all physical parameters (fields, stresses etc.). To simplify calculations, we assume that only vortex fields (Gernets et al., 2002) are emitted outside the piezocrystal, and do not take into account screening by environmental rocks. Hence we can use the well-known expressions for emitted EM waves (Landau, Lifshitz, 1967). The vortex character of the two sources allows us to approximately con- vert them to an expression for the magnetic field created by a variable magnetic dipole (Gernets et al., 2002):

$\boldsymbol{H}(\boldsymbol{r}, t)=\frac{1}{c^{2} r}\left[\left[\partial_{t}^{2} \boldsymbol{m}(t-r / c), \boldsymbol{n}\right] \boldsymbol{n}\right]$

where $r$ is the distance from the dipole $\boldsymbol{m}$ to an observation point, $\boldsymbol{n}$ is the unit vector of this direction, and $\boldsymbol{m}(t)$ is the magnetic moment created in whole piezo-magnetic crystal due to the cracking $\boldsymbol{m}(t)=\frac{1}{4 \pi} \int_{V} \boldsymbol{M}\left(\boldsymbol{r}^{\prime}, t\right) d V^{\prime}=\frac{1}{4 \pi \mu_{0}} \int_{V} q_{i j k} \boldsymbol{e}_{i} U_{j k}\left(\boldsymbol{r}^{\prime}, t\right) d V^{\prime}$, where $\boldsymbol{M}\left(\boldsymbol{r}^{\prime}, t\right)$ is the magnetization (in $T \cdot \mathrm{m} / \mathrm{H}$ ) and $\boldsymbol{e}_{i}$ are unit vectors, and $V \approx L^{3}$ is the volume of the finite cracked piezo-crystal. Assuming that the influence of the magnetic field on the strains is negligible we have obtained $U_{i j}=s_{i j k l} \sigma_{k l}$, where $s_{i j k l}$ is the rigidity tensor, which is the inverse of the elastic one.

Finally, after integration within the crystal volume we obtain:

$\boldsymbol{H}_{t}(\boldsymbol{r}, t) \approx \boldsymbol{H}_{0}(\boldsymbol{r})\left(f_{t}(\tau+0.5)-f_{t}(\tau-0.5)\right) \equiv \boldsymbol{H}_{0}(\boldsymbol{r}) F_{t}(\tau)$,

where the amplitude of the magnetic field is:

$\boldsymbol{H}_{0}(\boldsymbol{r})=\frac{q_{i j k} s_{j k l m} \sigma_{c r}}{8 \pi \mu_{0}} \frac{v_{c}^{2}}{c^{2}} \frac{\sqrt{a L}}{r}\left[\left[\boldsymbol{e}_{i}, \boldsymbol{n}\right] \boldsymbol{n}\right] \eta_{l m}$.

The unit tensor $\eta_{l m}$ describes the angle dependence of stresses into the cracked crystal, and the next integral describes the time dependence of the emitted magnetic field with $\tau=t v_{c} / L$ is dimensionless time

$f_{t}(\tau)=\tau \int_{0}^{1} \frac{d s}{\left(s^{2}+\tau^{2}\right)^{5 / 4}}=\frac{\operatorname{sign}(\tau)}{\sqrt{|\tau|}} \int_{0}^{|\tau|} \frac{d s}{\left(s^{2}+1\right)^{5 / 4}}$

This function is continuous because $f_{t}(\tau)_{\tau \rightarrow 0} \approx 1.0 \times \tau / \sqrt{|\tau|}$ and $f_{t}(\tau)_{\tau \rightarrow \infty} \approx 1.2 \times \sqrt{|\tau|} / \tau$.

Analogously, calculations for the unloading emitted EM waves give:

$\boldsymbol{H}_{u}(\boldsymbol{r}, t) \approx \boldsymbol{H}_{0}(\boldsymbol{r}) \sqrt{\frac{L}{a}}\left(f_{u}(\tau+0.5)-f_{u}(\tau-0.5)\right) \equiv \boldsymbol{H}_{0}(\boldsymbol{r}) F_{u}(\tau)$,

where $f_{u}(\tau)=1 / \cosh ^{2}(\tau)$. The time dependent parts in parenthesis of Eqs. (4) and (7) are plotted in the Fig. 1 where it is presented the dimensionless amplitude of magnetic field emitted during cracking of a piezo-magnetic crystal on dimensionless time $\tau=t v_{c} / L$ :

1. Radiation generated by the tip of crack $F_{t}(\tau)$,

2. radiation generated by the wave of unloading $F_{u}(\tau)$ at $L / a=1$.

First, it is clearly shown that the maximum field created by the crack tip (curve 1) occurs at time $\tau=0$ midway between entrance $(\tau=-1)$ and exit $(\tau=1)$ moments. Second, we can 
see that this emission starts much earlier than the crack entering into the piezo-crystal and finishes much later after the crack leaves. Therefore, sufficient stresses exist in the crystal for much longer than the time of cracking. The wave of unloading (curve 2) is more localized. Therefore, the corresponding emission is closely related to entrance and exit times. This emission is similar to two flashes of lightning when the crack enters into the crystal and exits from it.

\section{Intensity and frequency of EM-waves emitted from cracked piezomagnetic crystal}

Using a known expression (Landau, Lifshitz, 1967) for investigations of frequency dependence of the radiation intensity emitted into a solid angle element $d \Omega$, it is possible to obtain:

$\frac{d \mathrm{E}(\Omega, \omega)}{d \Omega d \omega} \equiv I(\Omega, \omega)=\frac{c}{4 \pi^{2}}|\boldsymbol{H}(\boldsymbol{r}, \omega)|^{2} r^{2}$,

where $\boldsymbol{H}(\boldsymbol{r}, \omega)$ is the Fourier component. For the emission of the unloading wave we obtain:

$I_{u}(\Omega, \omega)=\frac{c}{4 \pi^{2}}\left|H_{0} r\right|^{2} \frac{L^{2}}{v_{c}^{2}} \frac{L}{a}\left(\frac{2 \pi \tilde{\omega} \sin (\tilde{\omega} / 2)}{\sinh (\pi \tilde{\omega} / 2)}\right)^{2}$,

where $\tilde{\omega}=L \omega / v_{c} \equiv \omega \tau_{c}$ is the dimensionless frequency, and $\tau_{c}=L / v_{c}$ is the time of the crystal break. From Eq. (9) it follows that the intensity of radiation tends to zero $\sim \tilde{\omega}^{2}$ at low frequencies, and it decreases exponentially at high frequencies. The first maximum of the spectrum has been numerically found to be about $\tilde{\omega}_{1}^{u} \approx 1.14$. The amplitudes of the next maxima have the tendency to decrease exponentially. For example, we have $\tilde{\omega}_{2}^{u} \approx 6.96$, and the amplitude of this maximum is equal to about $1.7 \times 10^{-7}$ times of the first value. Hence, the most intensive EM emission generated by the unloading wave has the frequency $\omega \approx 1.14 / \tau_{c}=1.14 v_{c} / L$, which is determined just by the time of the crystal break.

The frequency dependence of the radiation intensity produced by the tip of the crack was analogously found from Eqs. (4) and (6), which are suitable for large values of $\omega$ :

$I_{t}(\Omega, \omega)=\frac{c}{4 \pi^{2}}\left|H_{0} r\right|^{2} \frac{L^{2}}{v_{c}^{2}}\left(4 \frac{\sin (\tilde{\omega} / 2)}{\sqrt{\tilde{\omega}}} \int_{0}^{\infty} \frac{\sin (s)}{\sqrt{s}} d s \int_{0}^{\arctan (\tilde{\omega} / s)} \sqrt{\cos (u)} d u\right)^{2}$.

By using Eq. (10) we found that at low frequencies the intensity increases as $\sim \tilde{\omega}^{3}$ and at high frequencies it falls down as $\sim \tilde{\omega}^{-1}$. The maxima of this spectrum have also been numerically investigated and they are localized about the points $\tilde{\omega}_{1}^{t} \approx 2.61, \tilde{\omega}_{2}^{t} \approx 9.19$, and in general $\tilde{\omega}_{n}^{t} \approx 2.61+2 \pi n$, where $n$ is an integer. Their amplitudes decrease proportionally to $\tilde{\omega}_{n}^{-1}$, so that several first harmonics of such an emission can be observed due to piezo-crystal cracking.

In the Fig. 2 the above dependencies are presented in the dimensionless variables: frequency $\tilde{\omega}$ and intensity

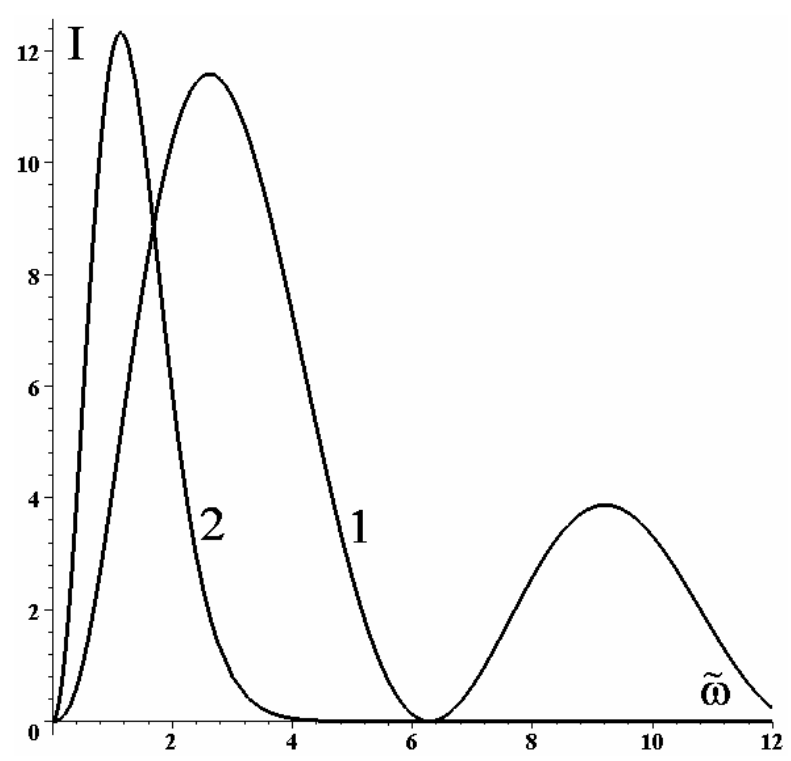

Fig. 2. Dependence of dimensionless emission intensity on dimensionless frequency: 1) radiation generated by the tip of crack, 2) the case of the wave of unloading at $L / a=7$.

$I \equiv I_{t}(\Omega, \omega) 4 \pi^{2} v_{c}^{2} / c\left|H_{0} r\right|^{2} L^{2}$. We also set $L / a=7$ to make similar scales of both intensities. In Fig.2 it is shown a dependence of dimensionless emission intensity on dimensionless frequency: 1) radiation generated by the tip of crack, 2 ) the case of the wave of unloading at $L / a=7$. The emission generated by the unloading wave has only one relatively narrow spectrum of frequencies.

\section{Discussion and conclusion}

For numerical estimations of $H_{0}$ we have used only maximum values of material tensor components for the magnetite crystal: $C_{11} \approx 2.86 \times 10^{11} \mathrm{~N} / \mathrm{m}^{2}, \quad q_{33} \approx 700 \mathrm{~N} / \mathrm{A} \times \mathrm{m}$, $\varepsilon_{33} \approx 0.09 \times 10^{-9} \mathrm{C}^{2} / \mathrm{N} \times \mathrm{m}^{2}, \mu_{33} \approx 1.57 \times 10^{-4} \mathrm{~N} \times \mathrm{s}^{2} / \mathrm{C}^{2}$ and $\rho \approx 5.15 \times 10^{3} \mathrm{~kg} / \mathrm{m}^{3}$ (Pan et al., 2001). Then the sound velocity is about $v_{s} \approx 7.45 \mathrm{~km} / \mathrm{s}$, inversion of the whole elastic tensor given the maximum value of the rigidity tensor component $s_{11} \approx 6.48 \times 10^{-12} \mathrm{~m}^{2} / \mathrm{N}$, and for the crack velocity, which is no more than the sound Rayleigh velocity we obtained the estimation $v_{c} \approx 0.85 v_{s}=6.3 \mathrm{~km} / \mathrm{s}$. We also assumed $\sigma_{c r} \approx C_{11}$ for the critical mechanical stresses, set $L \approx 10 \mathrm{~cm}$ for the crystal size, and assume that $\max \left(\left[\left[\boldsymbol{e}_{i}, \boldsymbol{n}\right] \boldsymbol{n}\right] \eta_{l m}\right)=1$. Since natural crystals inside rocks are not perfect, we assumed that the crack size scale is close to the crystal size; therefore, we set $a \approx L$. Then Eq. (5) gives $H_{0} r \approx 1.8 \mathrm{~mA}$, therefore at the distance of $r=1 \mathrm{~km}$ we obtain $H_{0} \approx 1.8 \mathrm{mkA} / \mathrm{m}$ and a magnetic induction $B_{0} \approx 2.3 \mathrm{nT}$. Modern magnetometer devices can easily detect such values. They can be also used for analyses and estimations of source properties of low frequency EM waves created due to natural or artificial geophysical activity. 
Acknowledgements. Authors are thankful to two anonymous referees for their useful suggestions, which allowed us to improve the quality of this paper.

Edited by: P. Fabian

Reviewed by: two anonymous referees

\section{References}

Biadzhi, P. F., Gershenzon, N. I., Zilpimiani, D. O., Mandzhgaladze, P. V., Pokhotelov, O. A., Sgrin'ya, V., and Chelidze, Z. T.: Influence of a magnetic field on mechanical properties of ionic crystals during their deformation, Sov. Phys. Solid State, 32(8), 1352-1354, 1990.

Gernets, A. A., Makarets, M. V., and Koshevaya, S. V.: Electromagnetic Emission Caused by the Fracturing of Piezoelectrics, Phys. Scripta, 65, 268-272, 2002.

Gershenzon, N. I., Zilpimiani, D. O., Mandzhgaladze, P. V., and Pokhotelov, O. A.: Electromagnetic radiation emitted by the apex of a crack during failure in LiF crystals, Doklady AN SSSR (Sov. Phys. - Doklady), 228(1), 75-78, 1986.

Gilder, S. A., LeGoff, M., Chervin, J., and Peyronneau, J.: Magnetic properties of single and multi-domain magnetite under pressures from 0 to $6 \mathrm{GPa}$, Geophys. Res. Lett., 31(10), L10612, doi:10.1029/2004GL019844, 2004.
Hayakawa, M. and Molchanov, O.A (Eds.): Seismo Electromagnetics, Lithosphere-Atmosphere-Ionosphere coupling, Terrapub, Tokyo, 2004.

Huang, J. H. and Kuo, W. S.: The analysis of piezoelectric/piezomagnetic composite materials containing ellipsoidal inclusions, J. Appl. Phys., 81(3), 1378-1386, 1997.

Landau, L. D. and Lifshitz, E. M.: Electrodynamics of Continuous Media, Nauka, Moscow, 1982.

Landau, L. D. and Lifshitz, E. M.: Classical Theory of Fields, Nauka, Moscow, 1967.

Pan, E.: Exact Solution for Simply Supported and Multilayered Magneto-Electro-Elastic Plates, ASME, J. Appl. Mech., 68(4), 608-618, 2001.

Petrov, N. and Brankov Y.: Modern Problems of thermodynamics, Mir, Moscow, 1986.

Unger, D. J.: Analytical Fracture Mechanics, Dover Publ. Inc., Mineola, N.Y., 2001.

Vainshtein, B. K.: Modern Crystallography, 4, Nauka, Moscow, 1982.

Wang, B. W. and Mai, Y. W.: Fracture of piezoelectromagnetic materials, Mechan. Research Commun., 31(1), 65-74, 2004. 